\title{
Sensemaking: Bringing Theories and Tools together
}

\author{
Nikhil Sharma
}

School of Information, University of Michigan, Ann Arbor Address: 1075

Beal Ave, Ann Arbor, Ml- 48103 nsharma@umich.edu

This work is an attempt to reconcile three separate but influential threads in study of sensemaking. The first two of these are theories from different domains, human computer interaction $(\mathrm{HCl})$ and social/organizational psychology. The last thread is that of design, of sensemaking support tools. Integrated, these three threads form a strong foundation for researchers, tool-designers and ultimately sensemakers themselves. Understanding and supporting the special role of people-people interaction can help us tie these separate threads together. This synthesis also suggests further research that can expand our understanding of sensemaking.

Introduction

Sensemaking often involves gathering information, gaining an understanding of the information and then using the understanding to finish a task. For example, sensemaking can occur when a person is trying to buy an unfamiliar product online or when a family is planning their vacation. It can occur when a research group is attempting to make sense of an emerging area or when an organization is trying to find strategies to deal with globalization pressures.

There are quite a few theories of sensemaking, with roots in fields including information science, $\mathrm{HCl}$ and social/organizational psychology. Due to their different roots, though these theories explicate the same phenomenom they are disparate and unconnected. This work attempts to reconcile two of these theories, that of Russell et al [6] and Karl Weick [9]. These theories were chosen not just for their influence but also because they are seemingly very different from each other. Reconciliation of these theories would expand their explanatory power and give us a richer understanding of sensemaking process.

Many tools for supporting sensemaking have also been designed by $\mathrm{HCl}$ and Human 
Information Interaction (HII) researchers and practitioners. These tools primarily help in gathering and organizing information. Yet many of these tools do not explicitly draw from sensemaking theories and rarely do theories receive feedback gained by the use of these tools. If major theories and tools could be reconciled this two-way communication between tools and theories could be opened up which would ultimately benefit both designs and theories.

Looking back at the examples given earlier, it is clear that sensemaking can occur at various levels of aggregation (individuals, small/large groups and even communities), often involving collaboration. Effective collaboration in sensemaking often involves attention to people-people interactions. It is these people-people interactions which can be used to reconcile Russell et al's model, Weick's framework and various designs of support tools.

\section{Russell et al's Sensemaking model}

Russell et al [6] proposed a model of sensemaking in which the cost structure of actions guided behavior. The theory, illustrating the use of representations, characterized sensemaking as "the process of searching for a representation and encoding data in that representation to answer task-specific questions". According to this theory, representations are chosen to lower costs related to various operations in sensemaking (finding data, encoding it \& using the encoded representations). Data that is not compatible with the current representation builds up as 'residue'. Residue represents elements relevant to the task that are not being handled properly. Too much residue creates a costly situation that may be worth trying to fix by finding a better representation or "shifting" the current representation somehow (see Figure 1). Representation shift is an important process in Russell et al's 'Learning Loop'. It is assumed that people will notice residue themselves and this residue will guide change. The next section provides many reasons why this might not be the case. 


\section{Learning Loop Complex}

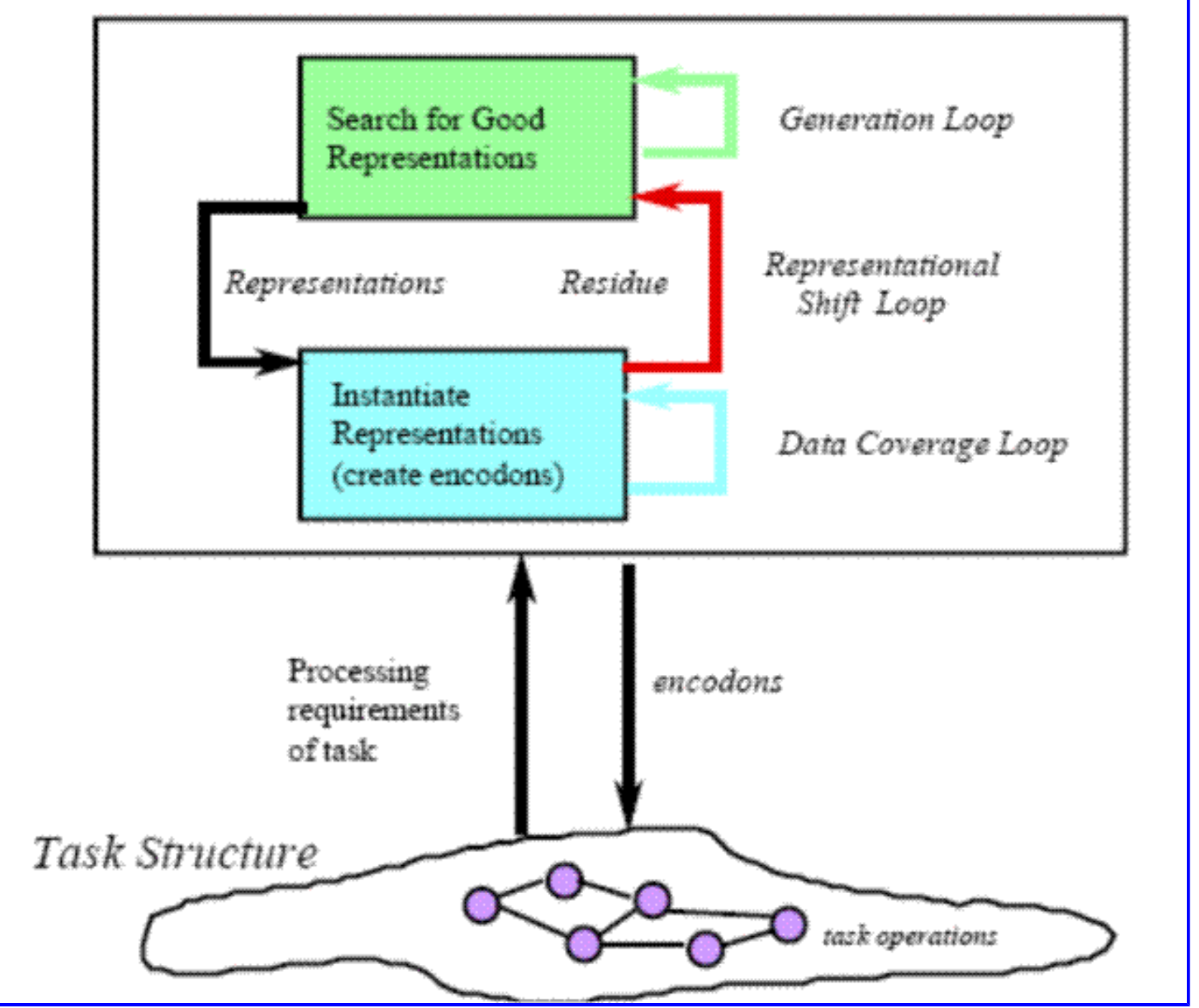

Figure 1. Russell et al's Sensemaking Model

\section{Weick's Sensemaking}

Karl Weick [9] takes a social psychology approach to sensemaking. According to Weick, sensemaking involves placement of stimuli into a framework. These frameworks could be categorizations, anticipations or assumptions. While characterizing sensemaking, Weick implies when done without particular care, sensemaking process is far from perfect.

First of these imperfections is our inability to shift representations easily due to the inertia of our representations. Weick points out that beliefs held strongly are not questioned so much, rather the focus is on confirming them. This often results in selective attention to cues that confirm beliefs. Compatible data increases confidence 
and incompatible data is just ignored. People may sometimes go to the limit of ignoring blaring warnings and cues, a process termed as 'fallacy of centrality' ("since I don't know about it- it must not be happening"). While inertia of frameworks and actions is helpful in a certain way because it helps continuity, it can be problematic in many cases. When the environment is dynamic and unpredictable, as is often the case in sensemaking, inertia and commitment can give people a false sense of security while they hold on to outdated frameworks. Another reason for ineffectiveness of sensemaking, according to Weick is our inability to find and use appropriate data. Often this extraction of information is based on existing frameworks and information channels sensemakers are accustomed to using. This means sensemakers are tuned mostly to information that is compatible with their representations and rarely do they come accross "residue".

It can be noted that the problems pointed out by Weick are in some ways related to our lack of ability in noticing residue and shifting representations easily. To get sensemaking on track Weick suggests tighter social connections and active communication channels for arguing, negotiation and updating. Moving sensemaking from an individual act to something that happens between people, Weick's recipe for effective sensemaking is centered on improving people-people interactions. It is this recipe of employing people-people interactions that can help us to expand Russell et al's model in useful ways.

\section{Reconciling Russell et al \& Weick's Sensemaking}

Figure 2 attempts to not only reconcile Weick and Russell et al's sensemaking frameworks but also to put people-people interactions in their deserving place within sensemaking framework. For this exercise Russell et al's model was expanded upon because of its crispness and straightforwardness. The placement of people-people interactions also highlights issues and opportunities of collaboration for sensemaking. 


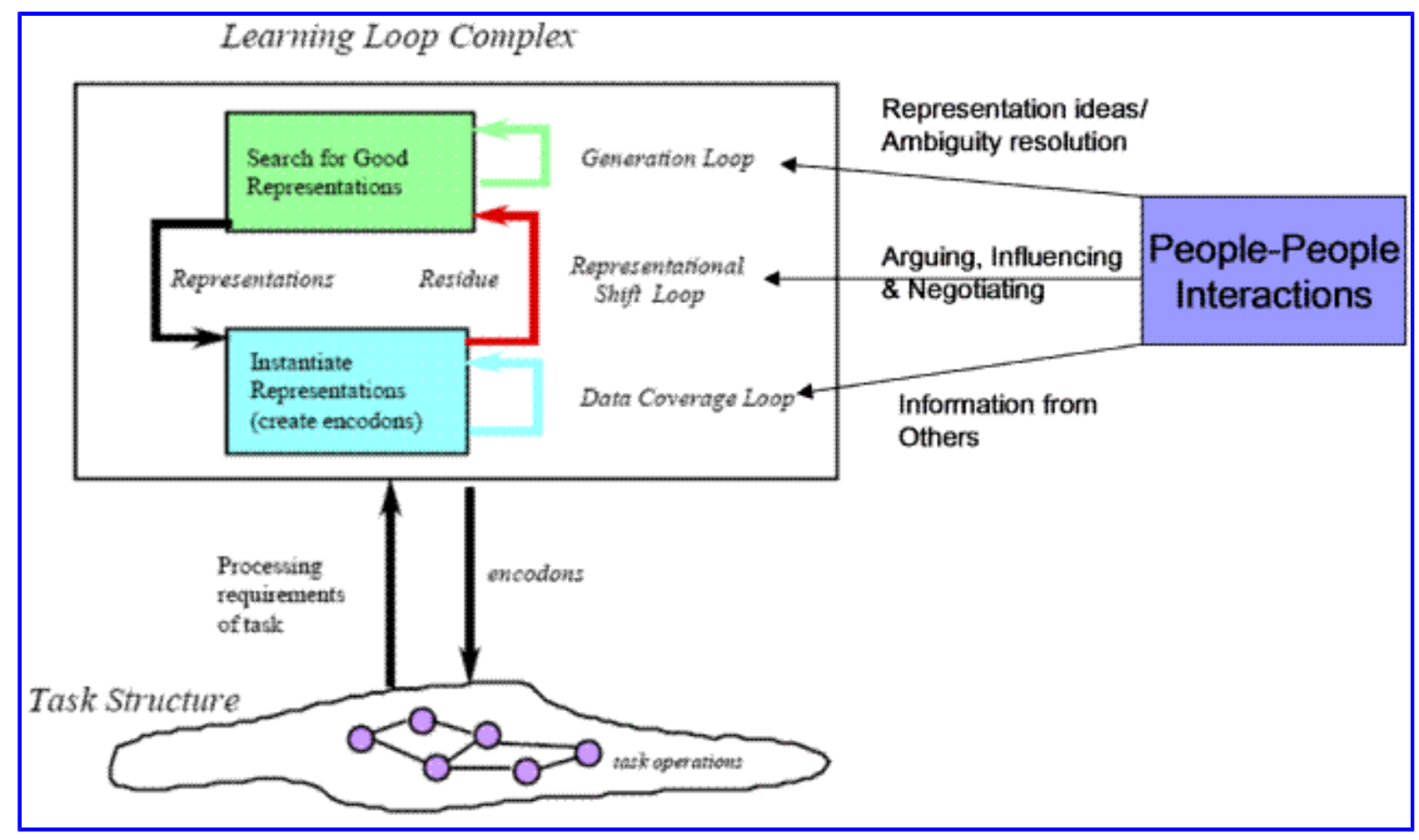

Figure 2. Expanded Russell et al's model with people-people interactions

Russell et al do not elaborate how representations are generated; this is the first opportunity for collaboration in sensemaking, other people might come in handy while generating representations. When starting with a blank slate we may ask around for representations or may be given a head start by others' suggestions. Weick pointed out that often people do not notice residue on their own and do not shift representations easily. This offers the second opportunity for collaboration. People-people interactions can cause representation shift through arguing/negotiation. Interacting with others is what often causes us to notice residue and change our frameworks. Finally, the "data coverage loop" also offers opportinity to collaborate, here other people can be information sources.

\section{Sensemaking Tools \& Theory}

Figure 3 places various sensemaking support tools within the expanded Russell et al's model. The list of tools is not exhaustive, nor is it implied that a tool exclusively fits in one slot in the framework; it is an attempt to point out the aspect of sensemaking that is most saliently supported by a particular tool. This exercise allows us to notice the relationship between various tools and the expanded Russell et al's framework. We can notice that some tools have focused on supporting sensemaking without paying particular attention to people-people interactions even though some of these tools were 
"multi-person tools". In Figure 3 these tools have been placed inside Russell et al's 'learning loop' as they have tried to aid either representation or information search. Zamir \& Etzioni [10] supported clustering of search results, which can be imagined to aid representation search while Hendry \& Harper [4] provided the user with a spatial canvas to develop representations.

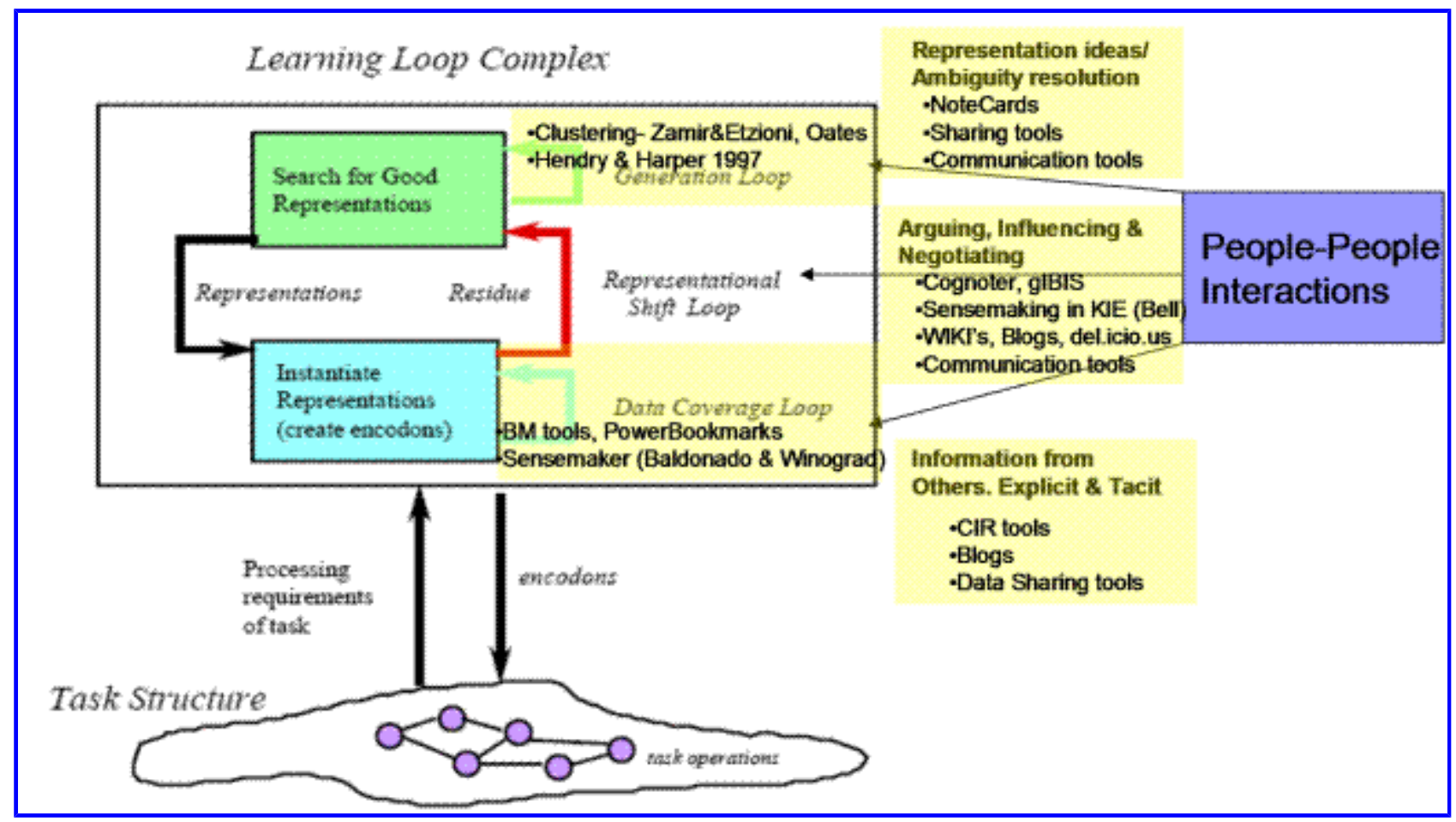

Figure 3. Expanded Russell et al's model with people-people interactions and Sensemaking tools (yellow highlights)

Tools supporting information collection and search include bookmarking tools like Li et al's [5] PowerBookmarks which have integrated querying and result saving as well as automatic classification. Sensemaker by Baldonado and Winograd [1] was also an information exploration tool that enabled search of multiple sources and bundled the results by domain, title or the author based on users specification. This information exploration tool also allowed progression in the search by expansion and replacement of terms, a feature that employed existing representations to guide data collection.

Some toolmakers have realized the importance of people-people interactions and have tried to support sensemaking by supporting these interactions. These tools are placed outside the 'learning loop complex' in Figure 3. Some of these tools like NoteCards [8] for example allowed people to develop and share representations. Other tools focus primarily on the role people-people interactions play in representation shifts. These include the Cognoter [7], gIBIS [3], Sensemaking in KIE tool [2] and various web tools like WIKI's, Blogs. Finally various tools have supported information collection from 
other people; these include various Collaborative Information Retrieval tools.

\section{Summary \& Next Steps}

The work so far brings two major but different sensemaking theories together using the various roles that people-people interactions play in sensemaking (provide representations, help modify them and provide information). These different roles also highlight the issues and opportunities for collaboration in sensemaking. The placement of tools within this framework can direct our choice of tools for different sub-activities of sensemaking and also presents directions for future tool design.

The next step in this research will be to examine in detail the various roles people-people interactions play in sensemaking. For representation generation, it may be worth exploring if representation generation tools like clustering are more helpful then interactions with other people during sensemaking? For representation shifting, we can explore how often people shift representations more while working alone vs. in a group? Lab studies offer an opportunity to take these next steps. Participants can be

given a sensemaking task similar to those mentioned in the introduction while working in groups or alone. Their representations can be tracked to see how the representations develop.

\section{Acknowledgements}

This work is funded by NSF grant IIS-0325347-ITR. The author is also grateful to George Furnas and Yan Qu and anonymous reviewers for their advice and help.

\section{References}

Baldonado, M.Q.W. \& Winograd, T. (1997) SenseMaker: An Information-Exploration Interface Supporting the Contextual Evolution of a User's Interests Proc. Conf. on Human Factors in Computing Systems 1997 11-18

Bell, P. (1997)

Using Argument Representation to Make Thinking Visible for individuals and Groups Proc. Conf. on Computer Support for Collaborative Learning, 1997

Conklin, J., Selvin, A., Shum, S. \& Sierhuis, M. (n.d.) Facilitated hypertext for collective sensemaking: 15 years on from gIBIS Proc. HYPERTEXT "01 Hendry, D. G. \& Harper, D. J. (1997) An Informal Information-Seeking Environment 
Li, W.S., Vu, Q. \& Chang, E.Y. (1999) PowerBookmarks: A System for Personalizable Web Information Organization, Sharing, and Management SIGMOD 1999 565-567

Russell, D. M., Stefik, M. J., Pirolli, P. \& Card, S. K. (1993) Cost structure of sensemaking Proc $\mathrm{CHI}$ "93

Stefik, M, Bobrow, D. G., Foster, G. Lanning, S. \& Tatar, D. (1987) WYSIWIS revised: Early experiences with multiuser interfaces ACM TRANS. OFFICE INFO. SYST 5(2), pp. 147-167

Trigg, R.H., Suchman, L.A. \& Halasz, F.G. (1986) Supporting collaboration in notecards Proc. CSCW

Weick, K. E. (1996) Sensemaking in organizations Newbury Park, CA: Sage Zamir, O. \& Etzioni, O. (1999) Grouper: a dynamic clustering interface to Web search results Proc. 8th International World Wide Web Conf. 1999 